\title{
El ejercicio de la Medicina y su entorno legal
}

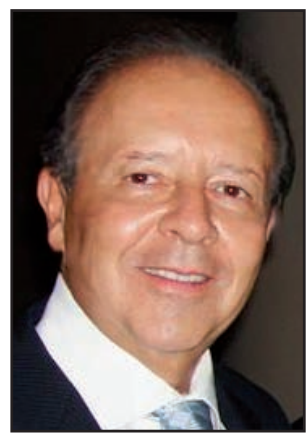

Fuente del Campo, $\mathrm{A}$.
Los casos de mala práctica médica surgen cuando un paciente es perjudicado o considera que fue perjudicado por un médico, enfermera u otro profesional de la Medicina, quien falló en indicar o aplicar el tratamiento adecuado para la atención de su salud.

La mal praxis o malapraxis médica se caracteriza porque quien proporcionó la atención actuó en forma negligente, imprudente o con impericia, implicando que esta persona no era competente o razonablemente hábil y perjudicando al paciente, incluso hasta provocar su deceso.

Diagnóstico y medicación erróneos representan un gran porcentaje de las reclamaciones en las demandas por negligencia médica. Cuando un médico falla en diagnosticar una enfermedad grave o bien no ha acertado al indicar el tratamiento y/o los medicamentos correctos, el paciente puede perder oportunidades de evitar complicaciones, daños colaterales y sus consecuencias.

Las demandas por negligencia médica han inducido la promulgación de leyes para atender diversos aspectos de estos problemas; esto aunado a la reacción de las compañías de seguros que mediante cifras topes mínimas / máximas y otros condicionantes intentan evitar el pago de indemnizaciones cuando se presenta una situación adversa a sus asegurados, ha generado la práctica de la "medicina defensiva". Esta y las demandas por responsabilidad médica han aumentado mucho los costos de la salud, obligando a desviar hacia el sistema legal una cifra importante de los escasos recursos con que cuenta el sector asistencial para la atención del paciente, la investigación y la formación de más médicos. El que el medico ejerza medicina defensiva depende más de su percepción de riesgo a ser demandado, que de otros factores.

\section{La responsabilidad médica}

El termino "responsabilidad" proviene del latín respondere, interpretado como "estar obligado". La obligación -obligatio- en el derecho romano clásico es la institución concebida como "el vínculo jurídico por medio del cual una persona-deudor- es comprometido frente a otra -acreedor- a cumplir con una determinada "prestación". La obligación implica dos elementos, débito y responsabilidad, el deber de cumplir con la prestación y la consecuencia derivada de su incumplimiento.

El prestador de servicios de salud tiene obligaciones que imponen deberes, qué hacer y qué no hacer. Cumplirlas tiene beneficios, no cumplirlas genera sanciones.

La "responsabilidad" es la consecuencia del incumplimiento, es decir, la obligación de rendir cuentas de los propios actos. Una profesión implica la capacidad calificada con que la persona a través de una actividad realiza su vocación dentro de un marco elegido, determinando su participación en la sociedad. Le sirve de medio de subsistencia y debe influir positivamente en la economía de la comunidad.

De esta forma, la responsabilidad profesional médica es la obligación que tienen los médicos y todo profesional de la Medicina de reparar y satisfacer las consecuencias de sus actos, omisiones y errores, voluntarios e involuntarios, cometidos en el ejercicio de su profesión.

El médico es sujeto de responsabilidad jurídica cuando actúa con negligencia, impericia y/o imprudencia.

Negligencia es el incumplimiento de los principios elementales inherentes a la profesión; esto es que sabiendo lo que se debe hacer, no lo hace, o a la inversa, que sabiendo lo que no se debe hacer, lo hace. Dicho de otra manera, existe cuando en determinada situación el

* Doctor en Medicina, Profesor Titular de Cirugía Plástica, Estética y Reconstructiva, Postgrado Universidad Nacional Autónoma de México de la División de Cirugía Plástica, Estética y Reconstructiva, Hospital General Dr. Manuel Gea González, México, DF. Miembro Emérito Academia Mexicana de Cirugía. Miembro Numerario Academia Nacional de Medicina de México. Coordinador Sub-Comité de Cirugía Plástica, Estética y Reconstructiva, Facultad de Medicina UNAM. Director Académico Asociación Mexicana de Cirugía Plástica Estética y Reconstructiva. Director Clínica de Cirugía Plástica Aqtuel, Ciudad de México, México.

** Doctora en Derecho, Profesora de Tiempo Completo (C), Facultad de Derecho, Universidad Nacional Autónoma de México. Miembro Sistema Nacional de Investigadores (SNI), Consejo Nacional de Ciencia y Tecnología (CONACYT) Nivel I, Ciudad de México, México. 
médico no actúa como lo haría cualquiera de sus colegas con los conocimientos y habilidades requeridas, en igualdad de circunstancias.

Impericia, es la carencia de las habilidades o conocimientos básicos, indispensables para ejercer determinada arte o profesión.

Imprudencia consiste en afrontar un riesgo sin haber tomado las debidas precauciones para evitar sus posibles consecuencias, procediendo con apresuramiento innecesario, sin considerar los inconvenientes que podrían resultar de esa acción u omisión. Imprudencia es el ir más allá de los límites razonables ${ }^{(1)}$.

\section{Responsabilidad penal, civil y administrativa}

La sanción por la comisión u omisión que sea calificada como delito puede consistir hasta en privación de la libertad y/o suspensión en el ejercicio profesional.

La responsabilidad civil se refiere a la obligación de reparar el daño causado a otro. Cuando surge de un obrar inadecuado o contario a lo establecido, se denomina "responsabilidad subjetiva". Cuando el daño resulta del uso de mecanismos, instrumentos, aparatos o sustancias peligrosas permitidos por la ley, se denomina "responsabilidad objetiva" o de "riesgo creado".

Cuando hablamos de responsabilidad civil del médico nos referimos a la obligación legal de la reparación económica del daño causado por una malapraxis. Conforme a la legislación sanitaria, la atención médica debe llevarse a efecto de acuerdo con los principios científicos y éticos con que la lex artis médica orienta su práctica. Si un prestador de servicios de salud causa algún daño a un paciente por no apegarse a la lex artis establecida para el caso concreto, incurre en responsabilidad civil.

La responsabilidad administrativa se inicia con una queja a partir de la cual integran el expediente del quejoso. Hasta este punto puede haber posibilidades de una conciliación. De no ocurrir, el procedimiento continuará, emitiéndose finalmente una resolución. La resolución puede liberar de responsabilidad al prestador de servicios de salud; en caso contrario, se emite una recomendación o una sanción: suspensión, destitución, inhabilitación temporal o definitiva y/o pago de daños y perjuicios. Tratándose de un servidor público es factible su inhabilitación temporal o definitiva para desempeñar empleos, cargos o comisiones en el servicio público, así como una posible sanción económica.

La responsabilidad médica es la obligación ética y legal del médico de responder por los actos que realiza en el ejercicio de su accionar profesional. El médico responsable lo es en la medida que asume plenamente el cuidado de su paciente, quien ha depositado su confianza en el al solicitar sus servicios profesionales. ${ }^{(2)}$

\section{Alcances legales en el ejercicio médico de la profesión.}

En el ejercicio de la Medicina cometer errores no está sujeto al perdón, como puede ocurrir con otras profesiones, ya que genera consecuencias en la salud y esta es uno de los derechos humanos fundamentales protegidos por la ley. El médico es sujeto de responsabilidad jurídica cuando actúa con negligencia, impericia y/o imprudencia.

También es necesario considerar el "error médico"; este es el daño provocado al paciente por la acción u omisión del médico al ejercer su profesión, sin cometerlo intencionalmente, conducta clínica incorrecta, aplicar un criterio equivocado, etc. y deriva en la obligación de responder por los daños ocasionados.

Es este tenor, es importante distinguir entre el error y la malapraxis médica, ya que el error puede ser excusable por no revestir la gravedad de la negligencia médica, condición que el Poder Judicial tiene bien claro.

Es importante señalar que tal y como expresa el juramento hipocrático, el médico no está obligado a garantizar la curación del paciente, sino al empleo de las técnicas adecuadas conforme al estado vigente de la ciencia médica y las circunstancias concurrentes en el caso. En México esto ha sido claramente asentado en la tesis con número de registro 2002/441 dictada por la Suprema Corte de Justicia de la Nación.

De igual forma, el médico cuenta con guías o protocolos expedidos por las autoridades sanitarias que marcan los criterios que debe considerar en el ejercicio de su profesión con el propósito de no incurrir en una posible malapraxis médica.

Los factores condicionantes de una demanda atribuibles al médico son: ${ }^{(3)}$

- Mala relación médico paciente.

- Aplicación superficial de procedimientos clínicos.

- Deficientes conocimientos y habilidades.

- Invasión de campos de la Medicina que no domina.

- Exceso de confianza.

- Pago por terceros.

En la actualidad, algunos médicos aplican los procedimientos de diagnóstico de manera superficial e incompleta; el interrogatorio exhaustivo detallado y la exploración física meticulosa, no son practicados por todos y subordinan esta responsabilidad a estudios de gabinete $\mathrm{y}$ procedimientos diagnóstico-terapéuticos sofisticados y costosos con el propósito explícito de evitar controversias y demandas posteriores. ${ }^{(4)}$

Otras causas atribuibles al médico son deficiencias en sus conocimientos, habilidades o destrezas por fallos en su preparación básica o de postgrado, con programas probablemente muy técnicos pero poco clínicos enfocados a la investigación pero desligados de la solución a los problemas cotidianos.

Es frecuente que algunos médicos, muchos de ellos sin el entrenamiento formal necesario, invadan campos o especialidades que no dominan, o bien que por exceso de confianza realicen procedimientos que rebasan su capacidad exponiendo al paciente, en el mejor de los casos, a complicaciones o postoperatorios largos, tortuosos y costosos, que en ocasiones comprometen su vida.

Un aspecto importante de la relación del paciente y su médico es la comunicación. Un importante número de inconformidades tiene su origen en la falta de información al paciente a propósito de su padecimiento, de su tratamiento y de su pronóstico. El consentimiento infor- 
mado pretende permitir que el paciente conozca los riesgos y beneficios de los procedimientos diagnósticos y terapéuticos propuestos, así como que acepte o rechace su realización, evitando muchas controversias.

Otro factor importante en el desempeño del médico, y causa de problemas, es el pago por terceros o medicina prepagada, como es la medicina institucional, las compañías de seguros y las administradoras de servicios de salud. Estos "pagadores" frecuentemente limitan al médico en la indicación de estudios de laboratorio y gabinete, consultas con especialistas y algunos tratamientos debido a su elevado costo o porque no cuentan con ellos, dando como resultado inconformidad del paciente.

La calidad de la atención puede verse comprometida por la ocurrencia de un error médico, definido como el acto cuyo resultado es una equivocación, no habiendo en él mala fe ni elementos de mala práctica. El error es la causa más frecuente de las consecuencias indeseadas de la atención médica, por encima de la malapraxis.

\section{La malapraxis médica}

Existirá malapraxis médica cuando se provoque daño en el cuerpo o en la salud de una persona física, sea este daño parcial o total, limitado en el tiempo o permanente, como consecuencia de una acción profesional realizada con imprudencia, negligencia o impericia en su profesión, o por no observar las normas de los deberes a su cargo, apartándose de la normativa legal aplicable. ${ }^{(5)}$

Existen diversos factores causales o de riesgo que

Tabla I. Tipos de errores que conducen a la malapraxis

\begin{tabular}{|c|c|}
\hline \multirow{4}{*}{ Diagnósticos } & Error o retardo en el diagnostico \\
\hline & Fallo en la indicación de pruebas pertinentes \\
\hline & $\begin{array}{l}\text { Fallo al actuar en base a los resultados de las } \\
\text { pruebas realizadas }\end{array}$ \\
\hline & Indicación de pruebas terapéuticas inadecuadas \\
\hline \multirow{5}{*}{ Terapéuticos } & Cuidado inapropiado o no indicado \\
\hline & $\begin{array}{l}\text { Error en el método o dosis de administración de } \\
\text { medicamentos }\end{array}$ \\
\hline & Error en la administración del tratamiento \\
\hline & $\begin{array}{l}\text { Realización errónea de cirugía, procedimientos } \\
\text { o pruebas }\end{array}$ \\
\hline & $\begin{array}{l}\text { Retraso evitable en tratamiento o respuesta a } \\
\text { pruebas anormales }\end{array}$ \\
\hline \multirow{3}{*}{ Preventivos } & Fallo por no indicar tratamiento profiláctico \\
\hline & Indicaciones profilácticas inadecuadas \\
\hline & Inadecuado seguimiento del tratamiento \\
\hline \multirow{3}{*}{ Otros } & Fallo en el equipo \\
\hline & Fallo en la comunicación \\
\hline & Fallo en otros sistemas \\
\hline
\end{tabular}

pueden conducir al error (Tabla I) pero algunos son perfectamente previsibles:

- Exceso de confianza, vacilación, timidez, rutina.

- Demoras en la atención.

- Inadecuada relación médico-paciente.

- Inadecuadas condiciones o elementos de trabajo.

- Falta de organización, disciplina y supervisión del cuerpo médico.

- Ausencia de reglas para la revisión de la calidad de la atención prestada.

- Problemas en la confección, custodia, conservación de historias clínicas.

- Falta de reglamentos que rijan el ejercicio básico de la especialidad.

Las causas de la malapraxis médica pueden resultar de las siguientes situaciones:

- Historia clínica mal confeccionada (la más frecuente).

- Profesional mal capacitado, poco actualizado, mal dormido y hasta mal alimentado.

- Interrogar al paciente y escucharlo atentamente es imprescindible para su diagnóstico y tratamiento.

- Mala relación médico-paciente (causa que generalmente motiva la queja).

- El marco de la relación debe ser cuidado por todos, cumplir la ley, definido por el profesional e identificado y respetado por el paciente, familiares y allegados.

El buen trato al paciente es parte del servicio del médico. Así, podemos encontrar 4 grandes grupos de faltas en la atención médica:

- Error de diagnóstico o elección terapéutica. Es fundamental considerar el sitio donde se presta la atención médica, circunstancias personales del profesional, causas o hechos que pudieron influir en el resultado.

- Faltas instrumentales o de técnica. Las lesiones o daños surgidos con el uso de equipos por fallas técnicas del operador o del propio equipo utilizado.

- Incapacidad para realizar adecuadamente una técnica, contribuyendo al daño o muerte del paciente.

- Falla en la identificación del paciente o bien del órgano enfermo. Deben delimitarse las responsabilidades de cada uno de los integrantes del equipo de salud. ${ }^{(6)}$

Las formas ocultas de la malapraxis:

- Maltrato por falta de equipamiento o equipamiento en mal estado.

- Maltrato por falta de honestidad del profesional.

- Falta de honestidad del paciente, familiar o responsable.

- Maltrato por reducción del tiempo de atención del paciente.

- Falta de registros o alteración de registros en la historia clínica.

- Maltrato al familiar del paciente o de la persona a cargo del mismo.

- Maltrato subsecuente al incumplimiento o mal cumplimiento del paciente a la prescripción. 
- Falsificación de drogas y medicamentos.

Los elementos principales para establecer malapraxis en casos concretos, son los siguientes:

- Sometimiento del paciente a riesgo innecesario.

- Agravamiento o muerte del paciente por falta de calidad en la atención.

- Presencia de lesiones injustificadas.

- Obtención de remuneraciones fraudulentas.

- Realización de prácticas o suministro de insumos notoriamente inapropiados.

- Presencia de actos de violencia física y/o moral.

- Incumplimiento de obligaciones, de medios, seguridad y en su caso, de resultados.

- Realización de actos médicos sin la previa validación de comisiones y/o comités de investigación y/o ética.

- Transgresión a normativas prohibitivas (ejemplo: aborto o eutanasia no permitidos por la legislación).

\section{La medicina defensiva}

El aumento de reclamaciones en el ámbito médico condiciona a los médicos a una actuación profesional de más seguridad, llevándolos a solicitar pruebas y procedimientos diagnósticos adicionales, a veces exagerados, con la intención de prevenir una demanda.

La medicina defensiva es el conjunto de conductas desarrolladas por los médicos para protegerse y evitar demandadas por malapraxis. Las demandas y juicios son la principal causa de este proceder defensivo del médico; en este sentido, la relación tradicional médico-paciente ha cambiado sustancialmente. El médico selecciona sus casos y rechaza o refiere a los que considera de riesgo, solicita múltiples estudios, frecuentemente innecesarios, para evitar reclamaciones por omisión o falta de evidencias para su diagnóstico y tratamiento. Realiza intervenciones quirúrgicas preventivas (innecesarias) o deja de hacer otras que son necesarias para evitar involucrarse con las implicaciones y posibles complicaciones de las mismas, quejas de sus pacientes, de los parientes y un posible litigio.(7)

En este contexto, las compañías de seguros han creado los llamados seguros de protección médica, con aumentos progresivos en la prima, contribuyendo al encarecimiento de la Medicina. La medicina defensiva se aparta de las normas éticas y es completamente ineficaz como estrategia de prevención de las reclamaciones de los pacientes porque no contribuye a lograr buena relación médico-paciente y agrega riesgos profesionales, como la realización de procedimientos médicos no indicados e innecesarios. La solución a los problemas de la medicina defensiva está en practicar una medicina asertiva, ejercida con amplio conocimiento de la profesión, sólido fundamento ético, accionar profesional y adecuado entorno médico-legal. ${ }^{(8)}$

Las causas del proceder defensivo del médico son múltiples:

- Falta de responsabilidad del médico.

- Proliferación de escuelas y facultades de Medicina.

- Avances en tecnología, comunicación e informática.
- Situación económica.

- Medicina institucional y administrada.

La medicina defensiva no es ética, pero no es el médico el único responsable de su origen y existencia. El médico se autoprotege al solicitar exámenes, prescribir medicamentos o realizar procedimientos quirúrgicos para evitarse problemas, pero este proceder inadecuado es consecuencia de su formación personal, tanto de su preparación deficiente o no actualizada como de su inadecuada formación ética y moral en el seno familiar, en la escuela y en su medio social. Mediante la medicina defensiva tiene la pretensión de protegerse ante una eventual futura reclamación del paciente. Se considera una forma no ética de ejercicio profesional desde que el acto médico deja de tener por objetivo central al paciente, trasladándose al propio médico. Tampoco es un ejercicio profesional éticamente admisible si se analiza desde la perspectiva de la salud pública, ya que agrega enormes costos al sistema de salud, sin justificación ni beneficios para los pacientes (Tabla II).

En este sentido, el médico está obligado a cumplir con lo que la ley y la Medicina señalan, por lo que su incumplimiento le obliga a responder ante su paciente. Entre las causas atribuibles al paciente como motivo de medicina defensiva. se enumeran los siguientes:

- Mayor cultura médica. El nivel educativo de las personas se ha incrementado, la población tiene acceso a los medios de comunicación masiva como la televisión, radio, periódicos, Internet, los cuales difunden noticias de nuevos medicamentos, procedimientos diagnóstico-terapéuticos y de casos de demandas contra médicos por supuesta negligencia, que les mantienen alerta y a la expectativa cuando acuden a consulta y más cuando tienen que internarse o someterse a estudios o intervenciones quirúrgicas.

Tabla II. Demandas legales al médico por su praxis

Factores Predisponentes

1. Número excesivo de prácticas y análisis invasivos.

2. Abogados que fuerzan el litigio para su propio beneficio.

3. Desconocimiento de la mayoría de los médicos de las normas legales que rigen su profesión, especialidad y el contrato de prestación de servicios con la entidad empleadora

Factores Desencadenantes

1. Industria del juicio a los médicos.

2. Reclamo de indemnizaciones excesivas.

3. Antecedentes de sentencias condenatorias simultáneas a médicos e instituciones de atención médica por altas sumas.

4. Facilidad para el demandante por "litigar sin costo"

Prevención y profilaxis

1. Buena relación médico/paciente

2. Consentimiento informado escrito, firmado y explicado al paciente y a un testigo.

3. Intervención activa del Comité de Ética Hospitalaria ante conductas médicas que pudieran implicar daño al paciente

${ }^{*}$ Fuente: Cuadernos de Medicina Forense. Año 2, No2, pp.73-78 
- El sistema prepago de servicios y seguros médicos. El hecho de que el paciente no seleccione a su médico predispone a la inconformidad; de este modo surgen controversias tanto en las compañías privadas de servicios de salud como en las instituciones médicas que administra el estado.

- La exigencia legítima de la sociedad a la atención médica oportuna, humanizada y de calidad genera reclamos inmediatos cuando no se cumplen sus expectativas y propicia la actitud defensiva del médico en su desempeño.

Los factores condicionantes del entorno social que influyen directamente son:

- Leyes más estrictas.

- Difusión inmediata y masiva de noticias médicas tanto positivas como negativas.

- Mal uso de la información,

- Demanda de atención médica oportuna, humanizada y de calidad.

\section{La responsabilidad médica y cómo determinarla}

La configuración de la responsabilidad médica en los tribunales se establece en base al concepto de lex artis ad hoc. En la doctrina del Derecho Sanitario, la lex artis ad hoc es entendida como el criterio de la correcta realización de un acto médico concreto, que es o será ejecutado por el profesional de la Medicina tomando en cuenta las características especiales de quien lo realiza, la complejidad y trascendencia vital del acto, así como factores endógenos, estado y participación del enfermo, de sus familiares o de la misma organización sanitaria, para calificar dicho acto de conforme o no con la técnica convencional requerida ${ }^{(9)}$ (Tabla III).

El acto médico se limita a las relaciones entre los profesionales médicos y el paciente. ${ }^{(10)}$

Para determinar la configuración de la responsabilidad

Tabla III. Responsabilidad médica

Dependerá del carácter del demandado, de las acciones que motiven el litigio y del tipo de responsabilidad que se pretenda reclamar

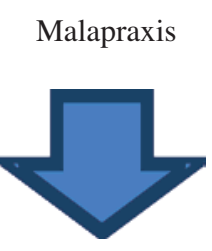

un objeto sancionador

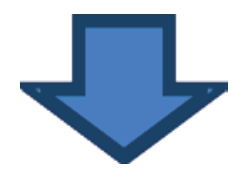

Responsabilidad administrativa y responsabilidad penal
Negligencia

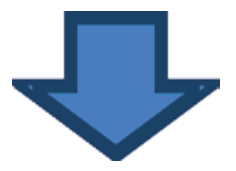

indemnización

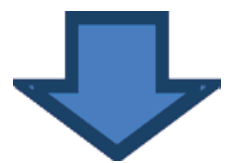

Responsabilidad
*Fuente: Suprema Corte de Justicia de la Nación,

https://www.scjn.gob.mx/Transparencia/Lists/Becarios/Attachments/192/RODRIGO\%20MONTES\%200CA.pdf por malapraxis médica se reconoce la necesaria intervención de expertos en la materia para determinar su existencia, dado que los jueces no poseen los conocimientos técnicos para dilucidar si un determinado profesional médico ha seguido las pautas que han establecido los miembros de la comunidad medica.

En México existen medios alternativos para la solución de conflictos suscitados entre el paciente y el profesional de la salud; sin embargo, estos no siempre son efectivos debido a que las partes no están obligadas a resolver su problema en estas instancias.

En Estados Unidos de América cuidan de que quienes participan en el estudio de las demandas sean médicos con las siguientes características:

- Deben poseer la habilidad /conocimiento promedio de los médicos.

- Deben tener el mismo grado de escolaridad y la misma escuela o tradición profesional que el acusado.

- Deben practicar su profesión en localidades similares.

En México, la Corte aún no cuenta con un desarrollo jurisprudencial amplio respecto a la responsabilidad médica, pero ha venido presentado algunos casos que permitirán desarrollar el concepto de lex artis ad hoc en un futuro. Sostienen que las guías o protocolos médicos elaborados por la Asociación Mexicana de Cirugía Plástica, Estética y Reconstructiva y los expedidos por la Secretaría de Salud responden a la cristalización escrita de criterios de prudencia que permiten habitualmente definir lo que se considera práctica médica adecuada y prudente en casos y procedimientos concretos, orientando la conducta diagnóstica y terapéutica aconsejable ante eventualidades clínicas especificas, lo que equivale a positivizar la lex artis. La Corte ha considerado que, desde el punto de vista jurídico, estas guías y protocolos proporcionan al médico un razonable amparo a la hora de justificar su actuación ante las reclamaciones de que puede ser objeto.

El aumento en los últimos años de las demandas por malpraxis, potenciado por los multifactores arriba señalados, ha preocupado al mercado de la salud, especialmente en los Estados Unidos de América, a tal punto que su evolución atenta contra la estabilidad del sistema y amenaza con colapsarlo. Son reclamos indemnizatorios que involucran cifras millonarias, aunque algunos no tienen fundamento causal ni judicial. Al respecto, ya hace años que las compañías aseguradoras han llegado a la conclusión de que el mercado de seguros de responsabilidad médica provoca pérdidas, igual que el de automotores, en virtud de su alta siniestralidad. Aun así, las modalidades de cobertura no satisfacen las necesidades de la demanda por la prescripción a los 10 años de los reclamos de esta índole.(11,12)

Aunque en México es escasa la información sobre los juicios y el error médico, en Estados Unidos de América son relativamente comunes, tal y como se desprende de una encuesta realizada a cirujanos especialistas quienes reportaron que más del 70\% de ellos habían sido deman- 
dados al menos una vez durante el ejercicio de su profesión. Estiman que 1 de cada 25 pacientes que han sufrido negligencia médica interpone una demanda; a pesar de ello, por cada 4 demandas entabladas 3 resultan con sentencia favorable para el médico tratante. ${ }^{(13)}$

\section{Estados Unidos y la medicina defensiva}

En Estados Unidos de América, el nacimiento de la medicina defensiva se relaciona con el fenómeno conocido como Malpractice Crisis, en que se ha observado un sustancial incremento en el número de demandas.

Muchos de los asegurados no tienen formación profesional adecuada, aumentando las posibilidades de tener problemas y ser demandados. El aumento de las sumas pagadas por compensación de los daños, así como el mayor número de demandas interpuestas, han repercutido en el precio del seguro de Malpractice medical, afectando a todos los médicos por igual. ${ }^{(14)}$ Según la Asociación Médica Mundial (AMM), en el mundo existe un aumento de la cultura del litigio que afecta de manera negativa la práctica de la Medicina, así como a la disponibilidad y calidad de los servicios de salud.

La cultura del litigio también ha dificultado la distinción entre negligencia y resultados adversos inevitables, lo que con frecuencia ocasiona la determinación aleatoria de la calidad de la atención. Esto ha contribuido a la percepción general de que cualquier persona puede demandar por casi cualquier cosa con la idea de obtener un gran premio. Esta práctica engendra cinismo y desconfianza tanto en el sistema médico como en el legal, con graves consecuencias para la relación médico-paciente. ${ }^{(15)}$

La AMM pide a las organizaciones profesionales nacionales que reaccionen exigiendo a sus legisladores las reformas necesarias para garantizar que las leyes y los sistemas jurídicos de sus países protejan a los médicos de los juicios sin fundamento, a la vez que se hagan garantes de promover un entorno médico-legal más adecuado que facilite ejercer una Medicina segura en beneficio de los pacientes. ${ }^{(16)}$

En Estados Unidos de América, para ganar una demanda por negligencia médica (medical malpractice) la persona lesionada debe probar que la atención médica recibida fue deficiente. En primer lugar los pacientes deben probar que existió negligencia médica, es decir, que el demandante debe demostrar que la atención médica proporcionada por el médico no cumplió con las normas pertinentes. En algunos casos esto no es difícil, como cuando por equivocación se realiza la cirugía en otra parte del cuerpo. En otros, demostrar la causalidad puede ser problemático, como en el caso de personas con enfermedades graves que podrían haber sufrido complicaciones de su patología incluso con la mejor atención médica. Identificar qué parte de la atención médica generó el problema puede ser un reto cuando participaron diversos médicos o proveedores en la atención. Por esta razón muchos tribunales tienen reglas especiales para hacer frente a situaciones en las que no es posible determinar con precisión los actos negativos que afectaron al paciente.
Por último, es necesario establecer la cantidad de dinero que debe ser otorgado a un demandante que gana. La aplicación de las reglas para determinar los daños puede ser complicada y debe tener en cuenta tanto las pérdidas reales económicas, tales como la pérdida de salarios y los costos de la atención médica en el futuro relacionados con la lesión, y las pérdidas no económicas, como el dolor, el sufrimiento o la pérdida de la compañía de un familiar, cónyuge o de un hijo. ${ }^{(17)}$

En un estudio publicado por la revista The New England Journal of Medicine, un grupo de médicos cualificados examinó una muestra aleatoria de 1452 demandas por negligencias de 5 compañías de seguros de responsabilidad civil que fueron resueltas entre 1984-2004. Una de las conclusiones más relevantes es que en casi el $40 \%$ de las demandas presentadas en Estados Unidos de América por malpráctice, no había error médico, es decir, eran infundadas. ${ }^{(18)}$

En México, de acuerdo con las estadísticas del 2017 de la CONAMED (Comisión Nacional de Arbitraje Médico), los médicos que fueron más demandados pertenecen al área de Ortopedia, a los que se dirigieron el $12.67 \%$ (249) de las demandas. Muy cerca les siguieron los cirujanos generales, a quienes fue dirigido el $10.3 \%$ (202) de las reclamaciones. Por su parte, el área de Obstetricia recibió el 7.8\% (153). El 6.16\% (121) de las demandas se dirigió a Ginecología, el 5.25\% (103) a las urgencias quirúrgicas, el 4.18\% (82) a la Neurocirugía y el $3.97 \%$ (78) fue dirigido a la Cirugía Plástica y Estética (Gráfico 1). ${ }^{(19)}$

El sistema de malpractice medical en Estados Unidos de América está basado en derecho de daños que apoya a los médicos responsables para no dañar a los pacientes intencionadamente o por negligencia. Si se considera el número de demandas y la frecuencia con que son levantadas, los médicos especialistas son los más frecuentemente afectados. ${ }^{(20)}$

Respecto al perfil de los demandantes: ${ }^{(21)}$ el $60 \%$ son mujeres; el $19 \%$ de las demandas se refieren a recién nacidos; el $12 \%$ son presentadas por mayores de 65 años; y 38 años es la edad media de los demandantes. El seg-

\section{Grafico 1}

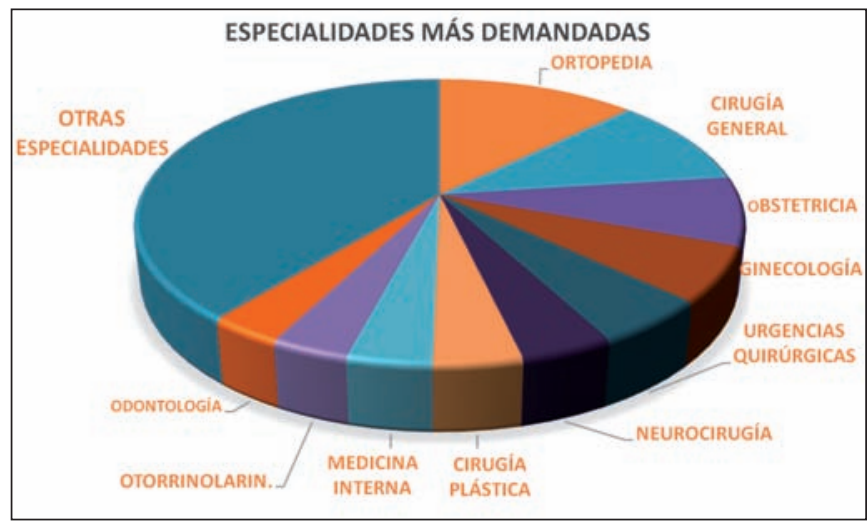

* Fuente:Aspectos de la nueva teoría de Estados Unidos y Reino Unido del reconocimiento de los errores médicos con efecto en la disminución de las demandas, M. Martínez León, D. Queipo Burón y C. Martínez León. 
mento de edad en el que se producen más reclamaciones es el comprendido entre los 35 y 49 años.

Dado el incremento de las demandas por malapraxis médica, al determinar si la causa fue un error cometido se pueden aplicar las llamadas "I'm sorry laws"(22). Estas implican la aceptación de los errores médicos sin que esta declaración sea prueba de culpabilidad. Estas leyes han mejorado la relación médico-paciente y la confianza en el profesional, ayudado a prevenir errores y han disminuido el número de reclamaciones y el costo legal de las mismas. ${ }^{(23,24)}$

\section{La medicina defensiva en México}

De acuerdo a CONAMED, en 2017 recibieron 3380 quejas. De estas, el $71.4 \%$ correspondieron al sector público mientras que la medicina privada, consultorios, clínicas y hospitales recibieron 969 inconformidades que representan el 28.6\%. ${ }^{(25)}$

La institución pública con mayores quejas fueron el Instituto Mexicano del Seguro Social (IMSS) con 1420, el Instituto de Seguridad Social de los Trabajadores del Estado (ISSSTE) con 679, los Hospitales Federales de la Secretaria de Salud con 100, y la Secretaria de Salud en la ciudad de México con 89, etc. En su reporte estadístico de 2017 la CONAMED reporta 15.256 asuntos recibidos y 15,878 concluidos. Dieron asesoría en 2481 casos,

Tabla IV. Quejas concluidas según especialidad médica y sector de atención

\begin{tabular}{|c|c|c|c|c|c|}
\hline \multirow[b]{2}{*}{ Especialidad Médica } & \multicolumn{5}{|c|}{ Sector involucrado } \\
\hline & Total & Público & $\begin{array}{l}\text { Asistencia } \\
\text { Social }\end{array}$ & $\begin{array}{l}\text { Seguridad } \\
\text { social }\end{array}$ & Privado \\
\hline Total & 1964 & 1429 & 6 & 1309 & 535 \\
\hline ACUPUNTURA & 1 & 0 & 0 & 0 & 1 \\
\hline ADMINISTRACION & 8 & 5 & 0 & 5 & 3 \\
\hline ALCOLOCIA Y CUNICA DEL DOLOR & 0 & 0 & 0 & 0 & 0 \\
\hline ANESTESIOLOCIA & 18 & 16 & 0 & 15 & 2 \\
\hline BIOLOCIA DE LA REPRODUCCION & 7 & 1 & 0 & 1 & 6 \\
\hline CARDIOLOGIA & 23 & 21 & 0 & 20 & 2 \\
\hline CIRUCIA ARTROSCÓACA & 2 & 2 & 0 & 2 & 0 \\
\hline CIRUCIA de CABEzA Y CuELLO & 3 & 3 & 0 & 3 & 0 \\
\hline CIRUCIA CARDIOTORÁCICA & 6 & 5 & 0 & 3 & 1 \\
\hline CIRUCIA DE CASTROENTEROLOCIA & 9 & 7 & 0 & 7 & 2 \\
\hline CIRUCIA ENDOSCOPICA & 5 & 2 & 0 & 1 & 3 \\
\hline CIRUCIA GENERAL & 158 & 131 & 0 & 116 & 27 \\
\hline CIRUGIA LAPAROSCOPICA & 5 & 1 & 0 & 1 & 4 \\
\hline CIRUCIA MAXILOFACIAL & 4 & 3 & 0 & 3 & 1 \\
\hline CIRUCÍA NEUROLÓGICA & 47 & 39 & 0 & 36 & 8 \\
\hline CIRUCIA PEDIATRICA & 13 & 12 & 0 & 11 & 1 \\
\hline CIRUCIA PLASTICA Y ESTETICA & 40 & 5 & 0 & 2 & 35 \\
\hline CIRUCIA RECONSTRUCTIVA & 25 & 23 & 0 & 19 & 2 \\
\hline CIRUCIA VASCULAR Y ANGIOLOCIA & 11 & 8 & 0 & 8 & 3 \\
\hline $\begin{array}{l}\text { COMUNICACIÓN, AUDIOLOCIAA, } \\
\text { OTONEUROLOCIA Y FONIATRIAA }\end{array}$ & 2 & 2 & 1 & 1 & 0 \\
\hline DERMATOLOCIA & 11 & 1 & 0 & 1 & 10 \\
\hline ENDOCRINOLOCIA & 6 & 6 & 0 & 5 & 0 \\
\hline ENDODONCIA & 16 & 1 & 0 & 1 & 15 \\
\hline ENDOSCOPIA & 4 & 4 & 0 & 4 & 0 \\
\hline ENFERMEDADES DEL COLON Y RECTO & 1 & 1 & 0 & 1 & 0 \\
\hline ENFERMERLA & 2 & 1 & 0 & 1 & 1 \\
\hline EPDEMIOLOCIA & 0 & 0 & 0 & 0 & 0 \\
\hline EXODONCIA & 12 & 2 & 0 & 2 & 10 \\
\hline GASTROENTEROLOGIA & 11 & 9 & 0 & 8 & 2 \\
\hline GENETICA & 0 & 0 & 0 & 0 & 0 \\
\hline GERIATRIA & 1 & 0 & 0 & 0 & 1 \\
\hline GINECOLOCIAA & 82 & 62 & 0 & 58 & 20 \\
\hline HEMATOLOCIA & 5 & 4 & 0 & 4 & 1 \\
\hline HOMEOPATIA & 2 & 0 & 0 & 0 & 2 \\
\hline IMPLANTOLOCIA DENTAL & 22 & 1 & 1 & 0 & 21 \\
\hline
\end{tabular}

1321 fueron de gestión inmediata y en otros solo emitieron un dictamen. ${ }^{(26)}$

En 2017 la Estadística de Quejas Concluidas apunta que en los casos de quejas contra médicos especialistas se emitieron 227 dictámenes, 175 de ellos por evidencias de malapraxis, 8 por Cirugía Plástica. De estos casos, 71 concluyeron con una decisión (laudo), 10 de ellas con responsabilidad institucional. En 28 de los casos se pudo comprobar la malapraxis, 20 del sector privado especialmente en casos de cirugía ( 2 de Cirugía Plástica) y 8 de medicina institucional. El 3.4\% de los casos (519) se resolvieron por conciliación, mientras que en en $6,25 \%$ (953), no se llegó al acuerdo. El 1.65\% (251) de todo el país fueron cerrados por "falta de interés procesal" de los presuntos agraviados.

La mayoría de las quejas está relacionada con 3 asuntos: mala relación médico-paciente, mala cirugía y mal diagnóstico, y con los servicios de especialidades médicas de Cirugía General y Ginecología. La Cirugía Plástica tiene pocos casos que hayan llegado a proceso, la mayoría se resuelven con la mediación de la CONAMED (Tabla IV).

En este contexto, la medicina defensiva surge como una respuesta de los médicos y prestadores de servicios de salud a la judicialización de los hechos adversos que se les pueden presentar a los largo de su vida profesional. Se calcula que en México se gastan casi 100.000 millones de pesos debido a los médicos que ejercen alguna modalidad de medicina defensiva, cifra que representa el $20 \%$ del presupuesto del sector salud en el país.

El presupuesto acumulado del ISSSTE y del IMSS, de la SSA, dedicado a la defensa de los litigios que se presentan es de casi 80.000 millones de pesos de un presupuesto total de 530.000 millones al año. Una de las causas del alto gasto es el tiempo que toma al Estado llegar a la resolución de los casos de este tipo, llegando a ser de hasta 12 años, cuando podrían acudir a instancias federales conciliatorias que facilitan el camino, como es la CONAMED.

\section{Conclusiones}

- Ante el aumento de demandas por negligencia y por mala praxis médica, los profesionales de la salud han buscado la aplicación de medidas para prevenir un conflicto médico legal, dando lugar a la medicina defensiva.

- La práctica de la medicina defensiva aumenta significativamente los costos de la atención médica, retrasa el estudio y tratamiento de los pacientes, en ocasiones hace inaccesible la medicina privada para las personas de bajos recursos, afecta a la capacidad económica de las instituciones para contar con los insumos necesarios, y limita la evolución de la ciencia médica.

- El fenómeno de la cultura de la demanda se ha globalizado; el incremento de las demandas coincide con el exagerado aumento de las sumas pagadas por compensación de daños que ha repercutido en 
el precio de las primas que anualmente pagan los médicos.

- Las demandas por la malapraxis médica han obligado a los profesionales de la Medicina a contratar un seguro contra estas eventualidades. Actualmente en muchos hospitales privados y en las instituciones asistenciales del país se le exige al médico contar con seguro de malapraxis para que pueda trabajar alli, ser contratado o aceptado como residente.

- La responsabilidad médica se deriva del actuar de un profesional, de la no observancia de un reglamento en el trabajo, de una infracción a un ordenamiento administrativo, o de una norma penal, cuyas consecuencias jurídicas van a ser distintas en cada uno de los casos.

- Para determinar la responsabilidad por malapraxis médica, los tribunales se basan en el concepto de lex artis ad hoc. También se acepta la participación de expertos en la materia (peritos), para determinar la existencia de malapraxis médica, ya que los jueces no tienen los conocimientos técnicos para determinar si un profesional médico ha seguido las normas establecidas por la comunidad médica.

- La ética médica demanda la disminución y la prevención del error. El ejercicio profesional del médico y de otros participantes implica riesgos, por ello no deben garantizarse resultados positivos al paciente para evitar que, de no ocurrir así, se de lugar a una demanda por negligencia médica.

\section{afuentedelcampo@prodigy.net.mx} alma_rios_r@hotmail.com

\section{Bibliografía}

1. Oscar, Garay. Treinta años de mala praxis médica, http://www.intramed.net/contenidover.asp?contenidoID=60662

2. Paredes Sierra, R. Medicina Defensiva, Pp.80-81, http://www.facmed.unam.mx/publicaciones/libros/pdfs/eeadlm.pdf

3. Cisneros Cortés SM. Límites jurídicos del arbitraje médico en México, Tesis de licenciatura. México, Facultad de Derecho y Ciencias Sociales Universidad Michoacana de San Nicolás de Hidalgo, Octubre 2009

4. Iraola LN. Apuntes Sobre la Responsabilidad Médica Legal y la Mala Praxis, Geo Salud, http://www.geosalud.com/malpraxis/malapraxis.htm

5. Bañuelo Delgado N. La mala práctica, CONAMED, http://www.conamed.gob.mx/comisiones_estatales/coesamed_nayarit/publicaciones/pdf/mala_practica.pdf

6. Paredes Sierra R. Ética y Medicina Defensiva, UNAM, $\mathrm{http} / / /$ www.facmed.unam.mx/sms/seam2k1/2003/ponencia_sep _2k3.htm
7. Rodríguez Almada H. De la medicina defensiva a la medicina asertiva, Revista de la Facultad de Medicina de la República de Uruguay, 2006, 3(22):167-168.

8. CONAMED: ¿Qué es la lex artis ad hoc? Disponible en: http://www.conamed.gob.mx/prof_salud/pdf/lex_artis.pdf.

9. Gore, A. Physicians, Surgeons, and Other Healers, https://advance.lexis.com/GoToContentView requestid $=8098 \mathrm{ad} 7 \mathrm{f}$ c846-a725-075cc98d35f541f0\&crid=19720d7f-9135-9b95-ba3 8 -e3bfbd7fcbc3.

10. Montes de Oca Arboleva R. Acto médico y responsabilidad profesional: reflexiones en torno a las diferencias entre las jurisdicciones mexicana y estadounidense, https://www.scjn.gob.mx/ Transparencia/Lists/Becarios/Attachments/192/RODRIGO\%20 MONTES\%20DE\%20OCA.pdf, p.18

11. Seoane M, Sotelo RA, Maccagno A. Los caminos del error médico, Cuadernos de Medicina Forense, Suprema Corte de Justicia de la Nación, Argentina, 2010, (2):73-78.

12. La información y el contenido multimedia, publicados por la Agencia de Noticias Andes. Pocos países suramericanos sancionan la mala práctica profesional, Ecuador avanza en el tema, http://www.andes.info.ec/es/noticias/pocos-paisessuramericanos-sancionan-mala-practica-profesional-ecuadoravanza-tema.html

13. Santamaría-Benhumea N, Garduño-Alanís A., et al. Aspectos legales del error médico en México, Rev. CONAMED, 2014, 19 (1): 32.

14. Martínez León M, Queipo Burón D.,et. al. Aspectos de la nueva teoría de Estados Unidos y Reino Unido del reconocimiento de los errores médicos con efecto en la disminución de las demandas, Revista de la Escuela de Medicina Legal, Universidad Complutense de Madrid, España, 2009:4.

15. Declaración de la AMM sobre la Reforma de la responsabilidad médica. Adoptada por la Asamblea General de la AMM, Santiago 2005.

16. Budetti P, et al. Medical Malpractice Law in the United States, Kaiser Family Foundation, USA, 2005:2-4.

17. Arimani Manso J. La medicina defensiva un peligro boomerang, Humanitas, 2007, 12:15

18. Martínez León M, Queipo Burón D, et. al. Medicina Lega una necesidad imperativa en México y en los Estados Unidos de América Op.Cit.:6-8.

19. Gallagher TH, Studdert D, Levinson W. Disclosing harmful medical error to patients. N England J Med 2007; 356 (26): 2713-2719.

20. Luce, JM. Medical malpractice and the chest physician, $\mathrm{Na}$ tional Institutes of Health, USA 2008, 134(5):1044-1050.

21. Studdert DM et al. Claims, Errors, and Compensation Payments in Medical Malpractice Litigation, $N$ Engl J Med 2006;354:2024-2033.

22. Momentum for special health courts is building. common good. Restoring common sense to America. En: http://commongood.org/search.html

23. Sanz L. La ética en la práctica. Cuando algo se hace mal: del error médico a la mala praxis, Traumatología Fundación MAPFRE 2008, 19(3):138-142.

24. Bown S. Sociedad de Protección Médica del Reino Unido. Medicalnewstoday. Diario Médico. 31 Oct 2008; Sección Normativa: página.http://www.diariomedico.com/edicion/diario_medico/normativa/es/desarrollo/1179800.html

25. Conamed recibió más de 2,000 quejas en 2014. El Economista, http://eleconomista.com.mx/sociedad/2015/06/07/conamed-recibio-mas-2000-quejas-2014.

26. En México gastan $\mathbf{1 0 0 , 0 0 0} \mathrm{mdp}$ en medicina defensiva. http://eleconomista.com.mx/sociedad/2011/10/19/mexico-gastan-100000-mdp-medicina-defensiva 